\title{
Delay in commencing treatment for MDR TB at a specialised TB treatment centre in KwaZulu-Natal
}

\author{
R Narasimooloo, A Ross
}

Background. According to the National Department of Health $(\mathrm{NDoH})$ guidelines, patients diagnosed with multidrug-resistant tuberculosis (MDR TB) must be referred to a specialised treatment centre for initiation of effective therapy. MDR TB is difficult to diagnose and the centralised referral model is beset with challenges that contribute to treatment delays, increased patient morbidity and mortality, and MDR TB nosocomial transmission. Culture and drug sensitivity testing (DST) takes 8 weeks or longer to obtain results while line probe assays (LPAs) can give a result in hours. LPAs and the GeneXpert MTB/Rif (GX) are ground-breaking discoveries for TB diagnosis. However, they are not easily accessible or available to those needing it, so culture and sensitivity testing remains the gold standard for diagnosis.

Aim. This study aimed to assess the delay in the initiation of MDR TB treatment and profiled the patients being referred to a specialised drug-resistant treatment centre in KwaZulu-Natal.
Results. Of all the patients, $75 \%$ referred showed a mean delay of 12.4 weeks from the date of sputum collection for culture and drug sensitivity testing to the start of treatment. Most of the patients were symptomatic for TB and HIV-positive.

Discussion. Our findings suggest that current policy on the initiation of effective treatment needs urgent revision. Staff should be appropriately trained in LPA and GX technology to reduce delays in initiating treatment for MDR TB. The NDoH's plans for rapid diagnosis and reducing the treatment burden on centralised MDR TB management facilities are in the early phases of implementation and will take years to achieve favourable and significant outcomes.

Conclusion. There is a significant delay in initiating definitive management for MDR TB.

S Afr Med J 2012;102(6):360-362.
South Africa has the highest incidence and mortality rates of tuberculosis (TB) in the world. ${ }^{1}$ Just under $2 \%$ of newly diagnosed $\mathrm{TB}$ and $6.7 \%$ of re-treatment $\mathrm{TB}$ patients have multidrug-resistant TB (MDR TB), ${ }^{2}$ translating into almost 7000 drug-resistant $\mathrm{TB}$ cases per year, with the highest proportion in 2008 from KwaZulu-Natal. ${ }^{1}$ MDR TB is defined as TB resistant to isoniazid and rifampicin. ${ }^{3}$ Failure of first-line TB regimens caused by poor adherence to drug-sensitive TB treatment is the most important factor in the development of MDR TB; the unavailability of effective TB drugs also contributes to this failure. ${ }^{3}$ As MDR TB symptoms and method of spread are the same as those of drug-sensitive TB, differentiation is impossible without further investigations. ${ }^{3}$ MDR TB can be identified by culturing sputum samples with drug sensitivity testing (DST) and by line probe assays (LPAs). Culture and DST take 8 weeks or longer, while LPAs can give a result in hours. ${ }^{2}$ The National Department of Health $(\mathrm{NDoH})$ guidelines require that patients diagnosed with MDR TB be referred to a specialised treatment centre for initiation of effective therapy. ${ }^{3}$ There are only a few such specialised centres located in South Africa's main cities. ${ }^{3}$ The $\mathrm{NDoH}$ recognises that centralised management of MDR TB faces challenges that cause treatment delays, increased patient morbidity and mortality, and MDR TB nosocomial transmission. ${ }^{4}$ We aimed to profile the patients being referred with MDR TB to a centralised treatment centre and investigate the time from the collection of sputum samples for DST to the initiation of effective therapy.

Department of Family Medicine, University of KwaZulu-Natal and Medical Research Council TB Unit, Durban

R Narasimooloo, MB BS

A Ross, MB ChB, MFamMed

\section{Methods}

The study hospital is a centralised treatment and referral centre for MDR TB in KwaZulu-Natal. The referral prerequisite is confirmation of MDR TB by culture and DST. Although LPAs are used, this has not been integrated into the $\mathrm{NDoH}$ treatment guidelines, and therefore a culture and DST is still the gold standard for diagnosis. ${ }^{3,5,6}$ Patients referred with results using tests other than culture and DST are assessed, and treatment is only initiated with confirmed results. ${ }^{3}$ Patients are admitted if they do not have a local clinic to receive their injections, are very ill or do not have social support to enable outpatient treatment. For outpatient treatment, a step-down facility or a local clinic close to home is required to supervise treatment. Both inpatient and outpatient facilities have long waiting lists of patients awaiting treatment.

We included all patients referred to the hospital in 2010 with a DST result confirming MDR pulmonary TB that qualified for treatment. Patients who presented with extrapulmonary MDR TB, mono- or polyresistant TB (not to isoniazid and rifampicin) and who did not present with results, were excluded. On average during 2010, 100 patients per month were initiated on treatment.

A sample size of 200 , representing $20 \%$ of the study population, was chosen. All the charts of patients initiated on treatment in 2 random months were analysed. Data from patient records and the hospital database were gathered on a data collation sheet and entered on a Microsoft Excel spreadsheet and analysed using the SPSS programme.

Descriptive analysis involved mean, standard deviation and range in normally distributed quantitative variables. As this was a descriptive study, no hypothesis was tested. The time interval (in weeks) between the collection of the sputum sample for culture and DST and the start of treatment was calculated. A delay was defined as the interval between sending sputum for DST testing and initiation of effective treatment exceeding 8 weeks; an acceptable range is between 6 and 8 weeks. ${ }^{3,5}$

Ethical approval was obtained from the Biomedical Research Ethics Committee of the University of KwaZulu-Natal and the Research Ethics Committee of the hospital where the study was conducted. 


\section{Results}

In total, 1158 patients were referred to the study hospital in 2010; 207 patient charts were reviewed of which 21 were excluded because they did not meet the inclusion criteria. Most patients were black, aged between 19 and 50 years, with an almost equal representation of men and women. Patients were mostly unemployed and symptomatic for TB with at least one previous TB episode. MDR TB contact history was poorly documented (Table 1). Of the patients, $69 \%$ were HIVpositive, on antiretroviral therapy (ART) $(75 \%)$ with CD4 counts $<250$ (59\%); 74 (40\%) were managed as inpatients, 101 (54\%) as outpatients, and $11(6 \%)$ were placed on the surveillance programme. This programme applies to patients referred with confirmatory DST results but who were not considered to have active MDR TB disease. Patients from the eThekwini municipality comprised $47 \%$, while $63 \%$ were from neighbouring districts; 111 (59.7\%) were on standard TB regimens at the time of referral, while 75 (40.3\%) were not on treatment. The mean delay from the date of collection of the sputum sample for culture and DST to initiation of treatment was 12.36 weeks (range 3 - 30 weeks) (Table 2).

\section{Discussion}

We aimed to investigate the time from the collection of sputum samples for DST to the initiation of effective therapy. A similar study

Table 1. Description of sample

\begin{tabular}{|c|c|c|}
\hline & Total $(N=186)$ & $\%$ \\
\hline \multicolumn{3}{|l|}{ Gender } \\
\hline Male & 97 & 52 \\
\hline Female & 89 & 48 \\
\hline \multicolumn{3}{|l|}{ Age } \\
\hline$<12$ years & 5 & 2.8 \\
\hline $12-18$ years & 17 & 9 \\
\hline 19 - 50 years & 145 & 78 \\
\hline$>51$ years & 19 & 10.2 \\
\hline \multicolumn{3}{|l|}{ Race } \\
\hline Black & 182 & 97.8 \\
\hline Indian & 3 & 1.6 \\
\hline White & 1 & 0.6 \\
\hline \multicolumn{3}{|l|}{ Employment status } \\
\hline Employed & 31 & 16.7 \\
\hline Unemployed & 155 & 83.3 \\
\hline \multicolumn{3}{|l|}{ MDR TB past exposure } \\
\hline MDR contact & 22 & 11.8 \\
\hline Occupational exposure & 3 & 1.6 \\
\hline Unknown & 161 & 86.6 \\
\hline \multicolumn{3}{|l|}{ Previous TB infection } \\
\hline No previous TB & 31 & 16.6 \\
\hline 1 episode & 71 & 38.1 \\
\hline 2 episodes & 63 & 33.9 \\
\hline 3 episodes & 19 & 10.2 \\
\hline$\geq 4$ episodes & 2 & 1.2 \\
\hline \multicolumn{3}{|l|}{ Symptomatic cough } \\
\hline No cough & 17 & 9.2 \\
\hline Cough & 169 & 90.8 \\
\hline \multicolumn{3}{|l|}{ HIV status } \\
\hline Negative & 47 & 25.2 \\
\hline Positive & 128 & 68.9 \\
\hline Unknown & 11 & 5.9 \\
\hline
\end{tabular}

Table 2. Time from the date of collection of sputum to start of treatment

\begin{tabular}{|c|c|c|c|}
\hline $\begin{array}{l}\text { Cumulative } \\
\text { weeks }^{*}\end{array}$ & $\begin{array}{c}\text { No. of months } \\
\text { delay (at 4-week } \\
\text { intervals) }\end{array}$ & $N=175$ & $\%$ \\
\hline $0-8$ & 0 & 44 & 25 \\
\hline $9-12$ & 1 & 54 & 31 \\
\hline $13-16$ & 2 & 42 & 24 \\
\hline $17-20$ & 3 & 21 & 12 \\
\hline $21-24$ & 4 & 7 & 4 \\
\hline $25-28$ & 5 & 5 & 2.9 \\
\hline $29-30$ & $>5$ & 2 & 1.1 \\
\hline \multicolumn{4}{|c|}{$\begin{array}{l}\text { *Time interval between the collection date of sputum sample for DST and start date of } \\
\text { treatment in weeks. } \\
\text { 'The everage expected time interval from collection of the sputum sample for culture and } \\
\text { DS and receivin a result is } 6-8 \text { weeks. For the purposes of this study, a delay is defined as a } \\
\text { a time period exceeding } 8 \text { weeks. }\end{array}$} \\
\hline
\end{tabular}

in Cape Town showed an average of 5 - 6 weeks' delay in initiation of treatment for patients with MDR TB. ${ }^{7}$ However, in our study 131 (75\%) of patients experienced delays of up to 22 weeks, and only $44(25 \%)$ patients were started on treatment without a significant delay. This is of concern, since almost $90 \%$ of all patients seen were coughing. Delaying treatment for patients who are actively coughing perpetuates the spread of MDR TB. It was previously believed that resistant strains of TB were not virulent enough for high transmission rates and that infection control was therefore not important. ${ }^{8}$ However, human-to-human spread of MDR TB is more common than anticipated, and the disease spreads while patients await diagnosis and initiation of treatment. ${ }^{8}$ In the Western Cape, $90 \%$ of all tested drug-resistant $\mathrm{TB}$ cases were smear-positive, suggesting that personto-person spread of MDR TB is a significant risk. ${ }^{9}$ Patients who are on ineffective drug regimens fuel TB drug resistance. ${ }^{3}$ South Africa has limited data on reasons for delays associated with the initiation of treatment for MDR TB, and more research is needed. Studies on drug-sensitive TB suggest that healthcare workers, systems and patient factors all contribute to delayed initiation of treatment. ${ }^{10,11}$ As part of the solution, the $\mathrm{NDoH}$ has suggested a decentralised model of MDR TB management; this may help to reduce the number of days between diagnosis and treatment initiation by increasing the number of treatment centres for referral, which will in turn reduce the transmission of disease. ${ }^{4}$ To meet this need, nurse-initiated treatment programmes, which are currently successful for HIV management throughout the world, are being strongly considered by the $\mathrm{NDoH}$ to integrate MDR TB and HIV management in communities. ${ }^{4}$

The World Health Organization (WHO) endorsed the use of LPAs for detecting TB and MDR TB, which decreases time to diagnosis from weeks to a day, and which requires highly trained technical staff. ${ }^{6}$ The GeneXpert MTB/Rif (GX) molecular test assay provides $\mathrm{TB}$ case detection and rifampicin-resistance testing with results being available from raw sputum in about 2 hours and does not require highly trained personnel. ${ }^{12}$ Availability of this test will enable the rapid detection of MDR TB for point-of-care management of patients who can be referred for treatment timeously. In 2009, the NDoH set a target to roll out 20 new secondary laboratory services by the end of 2010 to achieve rapid diagnosis of MDR TB. ${ }^{8}$ However, only 11 laboratories were established by the end of 2010. ${ }^{8}$ In March 2011, the $\mathrm{NDoH}$ approved 25 sites in South Africa's high-burden districts for GX to be installed; however, by the end of 2011, only 1 site had been fully capacitated. ${ }^{12}$ Lack of capacity at all sites for rapidly diagnosing 
MDR TB is compounded by the current policy of only treating MDR TB based on DST, regardless of the results from the GX. This policy completely negates the advantages of using the GX to diagnose MDR TB. Although documentation was poor, 22 patients (11.8\%) gave a history of a known MDR TB contact. There were 31 patients (16\%) with primary MDR TB (i.e. no previous TB history), with an incidence of $55 \%$ in women and $45 \%$ in men. The high number of primary MDR TB patients in the sample is of concern, as it is much higher than the $1.8 \%$ of the MDR TB population estimated by the $\mathrm{NDoH}$. Ours is a small sample but, if representative, it warrants further investigation. Based on this finding, healthcare workers must ensure that all high-risk patients presenting with $\mathrm{TB}$ are screened for MDR TB as per $\mathrm{NDoH}$ guidelines. ${ }^{3}$ In particular, HIV-positive patients with symptoms of TB need screening for MDR TB, as $71 \%$ of our patients with primary MDR TB were HIV-positive, with 59\% of them having a CD4 count $<250$. HIV-positive patients have an 8.4 times higher risk of harbouring MDR TB strains than HIV-negative patients. ${ }^{2}$ A small percentage of patients did not know their HIV status; a larger percentage of the HIV-positive patients did not know their CD4 count; and a significant number of HIV-positive patients were not on ART. This suggests that gaps remain in the South African HIV counselling and testing programme. All patients with MDR TB should be on ART, according to the $\mathrm{NDoH} \cdot{ }^{13}$

\section{Conclusion}

There is a significant time delay in the initiation of effective treatment for patients with MDR TB. Most of the patients who presented to the clinic were coughing, thereby fuelling the spread of community-transmitted MDR TB. LPAs and the GX are groundbreaking discoveries for TB diagnosis; however, they are not yet easily accessible or available to those in need of it. In view of the findings from this study, current policy on the initiation of effective treatment based on these results will need to be revised urgently. In addition, all staff should receive appropriate training if the newer technology is to reduce delays in initiation of treatment for MDR TB. The NDoH's proposed plans for rapid diagnosis and reducing the treatment burden on centralised MDR TB management facilities are all in the early phases of implementation, and it will take many more years to achieve a favourable and significant outcome.

Acknowledgements. We thank Doctors Cindy Narasimooloo, Laura Campbell and I Master, and Mrs Veronica Raman, Mr Sashin Moodliar and Ms Tonya Esterhuizen, for their inputs.

\section{References}

1. World Health Organization. Anti-tuberculosis Drug Resistance in the World. Report No. 4, 2008. WHO/HTM/TB/2008.394. http://www.who.int/tb/publications/2008/drs_report4_26feb08.pdf (accessed 11 March 2011).

2. World Health Organization. Multidrug and Extensively Drug-resistant TB: Global Report on Surveillance and Response. (2010). http://whqlibdoc.who.int/publications/2010/9789241599191_eng. pdf (accessed 11 March 2011).

3. National Department of Health. Management of Drug Resistant Tuberculosis. National Policy Guidelines. Pretoria: $\mathrm{NDoH}, 2010$

4. National Department of Health. Multidrug-resistant Tuberculosis. A Policy Framework on Decentralized and Deinstitutionalized Management for South Africa. Pretoria: NDoH, 2011. http:// www.doh.gov.za/docs/policy/2011/policy_TB.pdf (accessed 22 December 2011).

5. Loddenkemper R, Hauer B. Drug-resistant tuberculosis: a worldwide epidemic poses a new challenge. Dtsch Arztebl Int 2010; 107(1-2):9-10. [http://dx.doi.org/10.3238/arztebl.2010.0010].

6. World Health Organization. Molecular Line Probe Assays for Rapid Screening of Patients at Risk of 6. World Health Organization. Molecular Line Probe Assays for Rapid Screening of Patients at Risk of
Multidrug-resistant Tuberculosis. A Policy Statement. June, 2008. http://www.who.int/tb/laboratory/ Multidrug-resistant Tuberculosis. A Policy St

lpa_policy.pdf (accessed 22 December 2011).
7. Bamford CM, Taljaard JJ. Potential for nosocomial transmission of multidrug-resistant tuberculosis 7. Bamford CM, Taljaard JJ. Potential for nosocomial transmission of mult
(MDR TB) in a South African hospital. SAfr Med J 2010;100(7):438-441.

8. Institute of Medicine (IOM). The Emerging Threat of Drug-resistant Tuberculosis in Southern Africa: Global and local Challenges and Solutions (Summary of a joint workshop). Washington, DC: National Academy Press, 2011.

9. Johnson R, Warren G, van der Spuy N, et al. Drug-resistant tuberculosis epidemic in the Western Cape driven by a virulent Beijing genotype strain. Int J Tuberc Lung Dis 2010;14:119-121.

0. Chern JPS, Chen DR, Wen T. Delayed treatment of diagnosed pulmonary tuberculosis in Taiwan. BMC Public Health 2008;8:236 [http://dx.doi.org/10.1186/1471-2458-8-236].

11. Farah MG, Rygh JH, Steen TW, Selmer R, Heldal E, Bjune G. Patient and health systems delays in the start of tuberculosis treatment in Norway. BMC Infect Dis 2006;6:33 [http://dx.doi.org/10.1186/1471 2334-6-33].

12. National Health Laboratory Service. Gene Expert Implementation. http://www.nhls. ac.za/?page=genexpert_implementation\&id=69 (accessed 4 January 2012).

13. National Department of Health. South African HIV ART Guidelines. Pretoria: NDoH, 2010. 